\title{
Successful repair of total hand degloving injury by avulsed skin in situ replantation through vascular transplantation: a case report
}

This article was published in the following Dove Press journal:

Therapeutics and Clinical Risk Management

\author{
Yapeng Wang* \\ Yongjun Rui \\ Jiandong Zhou* \\ Fengfeng $\mathrm{Li}$ \\ Yongwei Wu \\ Jun Liu
}

Department of Hand Surgery, Wuxi No 9 People's Hospital Affiliated to Soochow University, Wuxi 214000,

People's Republic of China

*These authors contributed equally to this work

Correspondence: Yongjun Rui Department of Hand Surgery, Wuxi No 9 People's Hospital Affiliated to Soochow University, Lixi Rd. 999, Wuxi 214000,

People's Republic of China

Email wxswkyyryj@I63.com

\begin{abstract}
A 35-year-old man presenting with right-sided total hand skin degloving injury due to crushing of printing machine was reported in this study. The superficial vein of forearm was transplanted intraoperatively to reconstruct the blood supply, and the avulsed skin was replanted in situ. The patient was followed-up for 2 years, and satisfactory results and functional recovery were achieved.
\end{abstract}

Keywords: hand skin degloving injury, vascular transplantation, microsurgery, surgical skills

\section{Introduction}

Total hand skin degloving injury is associated with low morbidity in clinic; nevertheless, it remains a challenge in the surgical treatment of hands. It is frequently induced by huge shear force (Figure 1) and is characterized by the injury features of irregular wound margin and severe contusion. Generally, the avulsed skin, blood vessel, nerve, tendon, bone and joint are not on the same plane. There is severe intimal contusion, as well as artery and nerve avulsion in some cases. ${ }^{1-4}$ Therefore, the surgical treatment and rehabilitation of such diseases have posed a great challenge to surgeons.

Numerous scholars have long been trying many methods to repair the total hand skin degloving injury; however, the clinical demands can hardly be satisfied. Some new methods and technologies have emerged recently with the rapid development of microsurgery, among which treating total hand skin degloving injury with abdominal flap is simple and safe. Nevertheless, it is associated with postoperative flap swelling, which will lead to unsatisfying hand function. ${ }^{5,6}$ Moreover, anterolateral thigh flap combined with toe transplantation can also be applied in reconstructing the injured hand, but it will give rise to unfavorable hand function and appearance recovery. ${ }^{7}$ Çoban et $\mathrm{al}^{8}$ had reported a case with multiple digital degloving injuries treated with distal-based reverse forearm radial perforator flap, which represented a non-microsurgical treatment option. As a result, avulsed skin in situ replantation is the preferred choice for treating total hand skin degloving injury, which is linked with the superiorities of small wound, good hand appearance, no obvious scar contracture, elasticity, favorable sensation, and high patient acceptability. ${ }^{9}$ Briefly, such a technology has carried out vascular anastomosis through microsurgery, which frequently requires vascular transplantation in the presence of vascular defect.

In this study, a patient with total hand skin degloving injury successfully treated through avulsed skin in situ replantation is reported. To be specific, the superficial 


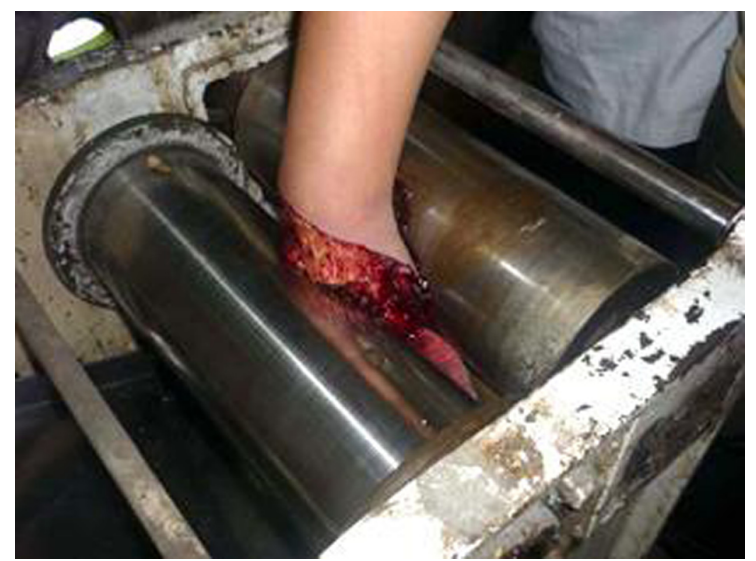

Figure I Total hand skin degloving injury caused by roller.

vein of forearm was transplanted intraoperatively; in the meantime, the arteria digitalis communis and ulnar artery were anastomosed. After surgery, the patient was followed-up for 2 years, and satisfactory efficacy and hand function had been attained, which were rarely reported in previous literature. Thus, the current study aimed to introduce our successful experience in this case.

\section{Ethics statement}

This study was approved by the Medical Ethical Committee of the Wuxi No 9 People's Hospital Affiliated to Soochow University. Written informed consent was obtained from the participant. The patient agreed to apply his photos and information to this medical research and allow his photos to be published in the journal of Therapeutic and Clinical Risk Management.

\section{Case report}

A 35-year-old man presented with right hand skin degloving injury due to crushing of printing machine. Skin degloving avulsion was seen in the right hand from the distal forearm to fingertip, while the thumb, maniphalanx, joint, tendon, nerve and intrinsic muscles remained relatively intact. Moreover, the degloved skin was basically intact; blood supply was available for the five fingers. The broken end of the common palmar digital arteries for the five fingers is located in the palm cross grain, while the vein of back of hand ruptured at the site $2 \mathrm{~cm}$ away from the carpal joint. Avulsion on hand back mostly began from the superficial layer of deep fascia, while avulsion on the palm side started from the superficial layer of palmar aponeurosis. In addition, skin avulsion on the fingers started from the superficial layer of flexor digital tendon sheath and extensor tendon (Figure 2A). Debridement and vessel-transplanting replantation were conducted in the patient under brachial plexus block, and six-hand veins were anastomosed intraoperatively (Figure 2B). Specifically, the superficial vein of forearm was transplanted, and the ruptured second to fifth arteria digitalis communis was anastomosed with ulnar artery, while the first to second arteria digitalis communis was anastomosed with radial artery (Figure 2C). Finally, the palmar and dorsal hand skin was slightly thinned, followed by pressure dressing. The whole operation time was $\sim 7 \mathrm{~h}$, and the surgery was operated by two groups of surgeons with over 10 years of microsurgery experience.

The flap had healed postoperatively, no sign of flap necrosis was seen, and the swelling had lasted for half a month. In addition, partial necrosis of palm skin was observed, which survived after change in dressing, and no catastrophic complication was observed. Rehabilitation training was initiated 10 days after surgery, and the swelling had gradually disappeared thereafter. The patient paid a subsequent visit 2 years postoperatively, and favorable texture of palmar and dorsal hand skin could be observed (Figure 3A and B). The final range of motion (ROM) is shown below. Active flexion of the first metacarpophalangeal joint of thumb was $\sim 60^{\circ}$, active flexion of the third to fifth metacarpophalangeal
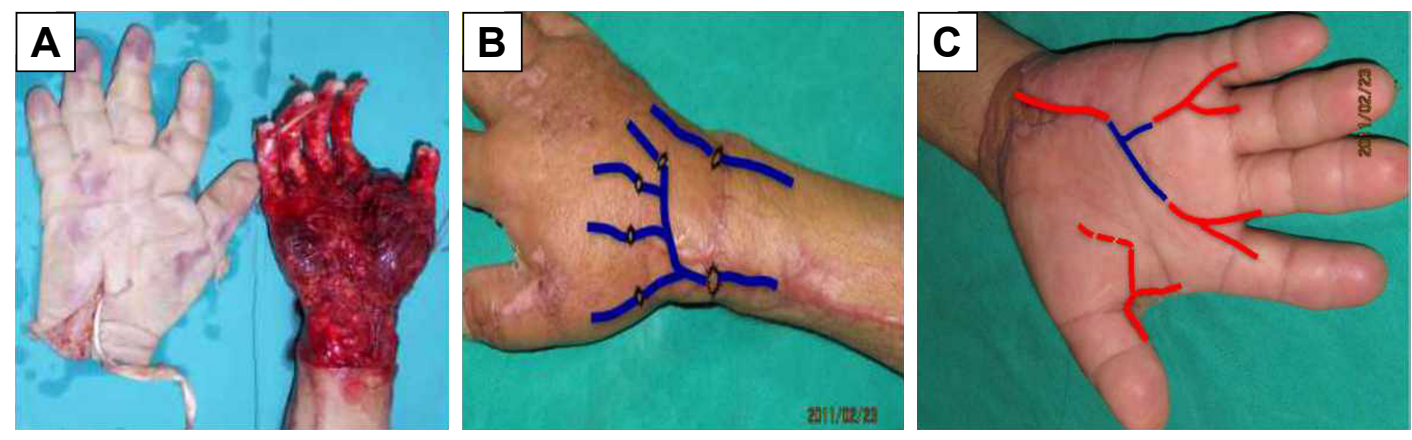

Figure 2 Total hand skin degloving injury.

Notes: (A) Before surgery. (B) Superficial forearm vein with four branches. (C) "Y shape" superficial forearm vein graft. 

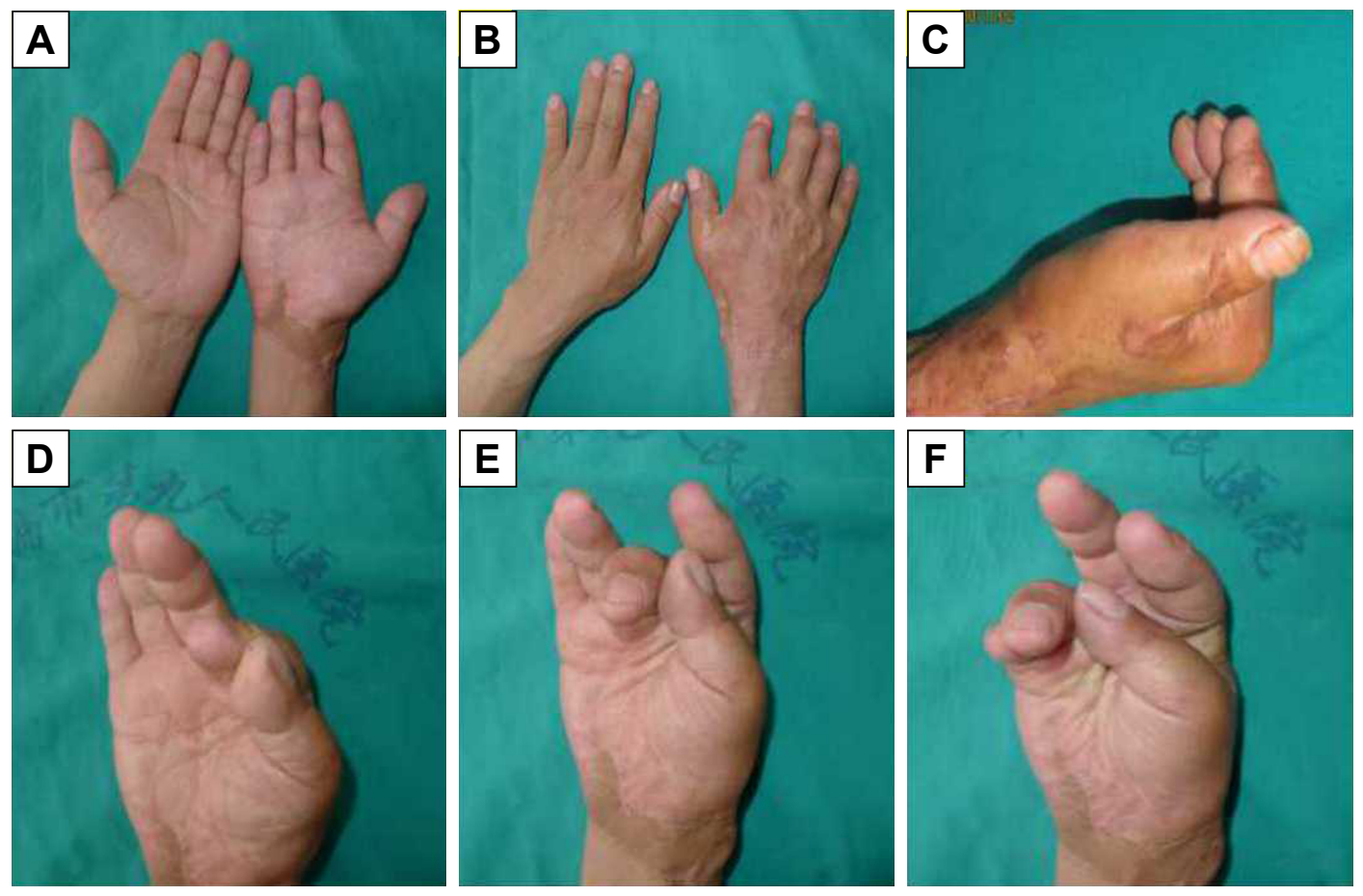

Figure 3 Appearance and function after surgery.

Notes: (A) Palm side at 2 years after surgery. (B) Dorsal side at 2 years after surgery.

(C-F) Finger flexion function at 2 years after surgery.

joints was $\sim 90^{\circ}$, and active flexion of second to fifth proximal interphalangeal point was $\sim 90^{\circ}$. Moreover, favorable finger appearance could be attained, thumb abduction and digital opposition function had been restored, and satisfactory recovery of finger flexion and extension function had been achieved (Figure 3C-F). We had anatomized the digital nerves for the five fingers intraoperatively, and the sensory function of these fingers had been restored to Grades S2-S3.

\section{Discussion}

Total hand skin degloving injury refers to degloving avulsion of hand skin and soft tissue as a result of external violence and roller crushing, which can be attributed to the protective conditional reflex and strong retraction of the body. Such an injury is characteristic of irregular wound margin and severe contusion. Generally, the avulsed skin, blood vessel, nerve, tendon, bone and joint are not located on the same plane. In addition, severe intimal contusion can be observed in some cases, along with artery and nerve avulsion. ${ }^{10}$ Typically, the skin superficial vein will be injured after total hand skin degloving injury. In the meantime, part of the skin is still connected to the finger root or proximal phalanx or tendon. However, the proper palmar digital artery is frequently subject to injury due to traction, which may manifest as vascular rupture or embolism. Therefore, it is completely possible to reconstruct the blood supply for the fingers once the distal and proximal ends of rupture can be found. Hand skin degloving injury is different from severed finger replantation, since cases undergoing severed finger replantation mostly have favorable finger blood circulation and integrity of the degloving skin with no obvious avulsion. In contrast, cases with total hand skin degloving injury generally suffer from incomplete skin integrity and severe contusion. Therefore, the skin condition should be determined first of all when treating total hand skin degloving injury using the vascular anastomosis in situ replantation technique. Mistaken judgment will result in not only replanted skin necrosis but also finger necrosis in some severe cases. Nevertheless, it is not always easy for accurate judgment of skin condition, which mainly depends on the experience of the operator. ${ }^{11}$

It is of high risk to treat total hand skin degloving injury with vascular anastomosis replantation. Nonetheless, it can lead to superior effects over other procedures in terms of hand appearance and function, as long as the skin has completely survived. In addition, such a surgery requires no massive skin donor site or additional donor sites, making it more easily acceptable among patients. Our experience from the successful surgical treatment for this case is that, first, thorough debridement is critical, since infection will inevitably affect skin survival and vascular patency. Particularly, 
a second debridement is necessary under microscope for severely polluted patients. Second, thorough intraoperative hemostasis should be performed, the drainage tube should be placed subcutaneously immediately after surgery, and smooth drainage should be guaranteed. Meanwhile, appropriate palmar and dorsal compression can avoid the formation of subcutaneous hematoma, which may affect skin survival or lead to infection. Typically, the pressure is appropriate not to compress the anastomosed vessel. Third, veins in the margin of palmar and dorsal hand avulsed skin should be anastomosed as far as possible, so as to reconstruct the venous return for the avulsed skin, which is one of the essential measures guaranteeing the survival of palmar and dorsal hand skin. Fourth, avulsed skin contusion should be carefully determined intraoperatively, and replantation should be avoided in cases with severe contusion, since non-survival of skin will definitely induce finger necrosis. Fifth, the arterial arch should be reconstructed in patients with superficial palmar arch avulsion and rupture in palm. Notably, the forearm dorsal reticular vein can be transplanted for reconstruction of the vascular defect, which is beneficial for the reconstruction of finger blood supply. Importantly, the problem of hand venous return pathway should be taken into account in the case of venous transplantation, and attention should be paid not to cut the major reflux vein. Notably, forearm dorsal superficial vein is appropriate for transplantation under this condition. As is shown in our case, the forearm dorsal superficial vein is transplanted, and the ruptured second to fifth arteria digitalis communis is anastomosed with ulnar artery, which facilitates the smooth recovery of hand appearance and function after replantation. Last but not the least, part of skin margin stitch can be removed to gently squeeze out the hematocele or blood clot, when subcutaneous hematocele is found in palm and dorsal hand during postoperative dressing change, followed by pressure dressing and fixation.

The integrity of vascular network should be ensured preoperatively, which is beneficial for the surgical planning and vascular reconstruction. In our case, we have overturned the avulsed skin before surgery and discovered that the broken end of the common palmar digital artery for the five fingers is located in the palm cross grain (Figure 2C). Thus, the arterial broken end for vascular grafting and anastomosis can therefore be found. Undoubtedly, blood supply is a key observational index after skin replantation, which can be judged based on the following aspects: 1) Observation of the replanted skin color: skin color is the most easily observed and most reliable objective index. However, the interfering factors should be avoided in observation, such as the influence of light and skin disinfector. Typically, the hot lamp can be deviated or turned off, so as to observe the flap color under natural light. Moreover, no disinfector such as iodine can be applied in coating the flap. Typically, a bright red color of flap indicates favorable blood supply, while a light or pale flap color is suspicious of arterial spasm or embolism. Meanwhile, a cyanosis or dark red color of flap reveals obstruction of venous reflux. 2) Capillary filling test: in this, the flap is gently compressed using the little finger pulp or cotton swab. Under general condition, the compressed flap is pale, which becomes ruddy 1-2 s after compression removal. It is noteworthy that the slow or disappeared filling reaction suggests insufficient or creased arterial blood supply, while the rapid filling indicates great possibilities of venous crisis. 3) Measuring skin temperature at regular time and location: skin temperature should be measured every hour within 3 days after surgery, which is then compared with that in the healthy side. Importantly, the location for measuring skin temperature should be fixed. Subsequently, skin temperature can be measured every $2 \mathrm{~h}$ on day 3 to day 5 and every $4 \mathrm{~h}$ on day 6 and day 7 . Temperature will not be measured after 7 days if no abnormality occurs. However, in the case of blood circulation disorder of flap, the skin temperature should be measured every hour. Specifically, the flap temperature lower than the healthy side by $>3{ }^{\circ} \mathrm{C}$ accompanying with color change usually reveals blood circulation disorder, which requires urgent management. 4) Flap swelling degree: the swelling degree can be determined according to the plica. Normally, plica can be observed in full and elastic flap, which disappears in the presence of excessive tension, along with cyanosis skin color, bright swelling, and subcutaneous vesicle. Mild inflammatory response and mild swelling can be observed after normal skin replantation, which may result from surgical trauma and will gradually fade away 3-7 days after surgery. However, the dressing should be checked to see whether it is too tight. Meanwhile, whether there is subcutaneous hematoma compression should also be checked if flap swelling is observed. Under such circumstances, the stitch can be intermittently removed to eliminate the hematocele and hematoma.

\section{Conclusion}

Repairing the total hand skin degloving injury with avulsed skin in situ replantation through vascular transplantation can completely repair the hand wound, in the meantime of attaining more favorable hand appearance and function. It can reduce injury in the donor site, shorten the course of therapy, and alleviate patient sufferings, thus attenuating the physical 
and mental burdens on patients. Therefore, it is an alternative method to treat total hand skin degloving injury.

\section{Disclosure}

The authors report no conflicts of interest in this work.

\section{References}

1. Hsu WM, Wei FC, Lin CH, Chen HC, Chuang CC, Chen HT. The salvage of a degloved hand skin flap by arteriovenous shunting. Plast Reconstr Surg. 1996;98(1):146-150.

2. Thomas BP, Katsarma E, Tsai TM. Replantation of total degloving of the hand: case report. J Reconstr Microsurg. 2003;19(4):217-220.

3. Rodriguez-Lorenzo A, Lin CH, Lin CH, Ching WC, Lin YT. Replantation of a degloved hand with added arteriovenous anastomoses: report of two cases. J Hand Surg Am. 2009;34(10):1864-1867.

4. Lo S, Lin YT, Lin CH, Wei FC. A new classification to aid the selection of revascularization techniques in major degloving injuries of the upper limb. Injury. 2013;44(3):331-335.

5. Pradier JP, Oberlin C, Bey E. Acute deep hand burns covered by a pocket flap-graft: long-term outcome based on nine cases. J Burns Wounds. $2007 ; 6: \mathrm{e} 1$
6. Nazerani S, Motamedi MH, Nazerani T, Bidarmaghz B. Treatment of traumatic degloving injuries of the fingers and hand: introducing the "compartmented abdominal flap". Tech Hand Up Extrem Surg. 2011; 15(3):151-155.

7. Senda $\mathrm{H}, \mathrm{Muro} \mathrm{H}$, Terada S, Okamoto $\mathrm{H}$. A case of degloving injury of the whole hand reconstructed by a combination of distant flaps comprising an anterolateral thigh flap and a groin flap. JReconstr Microsurg. 2011;27(5): 299-302.

8. Çoban YK, Öcük Ö, Bekircan K. Wrapping degloved fingers with a distal-based radial forearm perforator flap: a repair method for multiple digital degloving injury. Ulus Travma Acil Cerrahi Derg. 2017; 23(6):525-527.

9. Adani R, Castagnetti C, Landi A. Replantation of degloved skin of the hand. Plast Reconstr Surg. 1998;101(6):1544.

10. Kitidumrongsook P, Patradul A, Pataradool K. Resurfacing the degloved thumb up to the interphalangeal joint level with twin extended neurovascular island flaps. $J$ Hand Surg Br. 2006;31(5):562-565.

11. Ozaksar K, Toros T, Sügün TS, Kayalar M, Kaplan I, Ada S. Finger replantations after ring avulsion amputations. J Hand Surg Eur Vol. 2012; 37(4):329-335.
Therapeutics and Clinical Risk Management

\section{Publish your work in this journal}

Therapeutics and Clinical Risk Management is an international, peerreviewed journal of clinical therapeutics and risk management, focusing on concise rapid reporting of clinical studies in all therapeutic areas, outcomes, safety, and programs for the effective, safe, and sustained use of medicines. This journal is indexed on PubMed Central, CAS,

\section{Dovepress}

EMBase, Scopus and the Elsevier Bibliographic databases. The manuscript management system is completely online and includes a very quick and fair peer-review system, which is all easy to use. Visit http://www.dovepress.com/testimonials.php to read real quotes from published authors.

Submit your manuscript here: http://www.dovepress.com/therapeutics-and-clinical-risk-management-journal 